\title{
Extinction and Nonextinction for the Fast Diffusion Equation
}

\author{
Chunlai Mu, ${ }^{1}$ Li Yan, ${ }^{1,2}$ and Yi-bin Xiao ${ }^{3}$ \\ ${ }^{1}$ College of Mathematics and Physics, Chongqing University, Chongqing 400044, China \\ ${ }^{2}$ College of Elementary Education, Chongqing Normal University, Chongqing 400047, China \\ ${ }^{3}$ School of Mathematical Sciences, University of Electronic Science and Technology of China, Chengdu, Sichuan 610054, China
}

Correspondence should be addressed to Li Yan; yanli@163.com and Yi-bin Xiao; xiaoyb9999@hotmail.com

Received 28 February 2013; Accepted 28 April 2013

Academic Editor: Ru Dong Chen

Copyright (C) 2013 Chunlai Mu et al. This is an open access article distributed under the Creative Commons Attribution License, which permits unrestricted use, distribution, and reproduction in any medium, provided the original work is properly cited.

This paper deals with the extinction and nonextinction properties of the fast diffusion equation of homogeneous Dirichlet boundary condition in a bounded domain of $R^{N}$ with $N>2$. For $0<m<1$, under appropriate hypotheses, we show that $m=p$ is the critical exponent of extinction for the weak solution. Furthermore, we prove that the solution either extinct or nonextinct in finite time depends strongly on the initial data and the first eigenvalue of $-\Delta$ with homogeneous Dirichlet boundary.

\section{Introduction}

In this paper, we deal with the following fast diffusion equation with gradient absorption terms:

$$
\begin{gathered}
u_{t}=\Delta u^{m}+\lambda|\nabla u|^{p}, \quad(x, t) \in \Omega \times(0, \infty), \\
u(x, 0)=u_{0}(x), \quad x \in \Omega, \\
u(x, t)=0, \quad x \in \partial \Omega \times(0, \infty),
\end{gathered}
$$

where $0<m<1, p>0, \lambda>0, \Omega \subset R^{N}$ with $N>2$ is an open bounded domain with smooth boundary, and $u_{0} \epsilon$ $L^{\infty}(\Omega) \cap W_{0}^{1, p}(\Omega)$ is a nonzero positive function.

Equation (1) appears in a lot of applications to describe the evolution of diffusion processes, in particular, fast diffusion for $0<m<1$. In combustion theory, for instance, the function $u(x, t)$ represents the temperature, the term $\Delta u^{m}$ represents the thermal diffusion, and $\lambda|\nabla u|^{p}$ is a source.

Extinction and nonextinction are important properties for solutions of many evolutionary equations, especially for fast diffusion equations. In 1974, Kalashnikov [1] considered the Cauchy problem of equation $u_{t}=\Delta u-u^{p}$ and firstly introduced the definition of extinction for its solution; that is, there exists a finite time $T>0$ such that the solution is nontrivial for $0<t<T$, but $u(x, t) \equiv 0$ for all $(x, t) \epsilon$ $\Omega \times(T, \infty)$. In this case, $T$ is called the extinction time.
Since then, many authors became interested in the extinction and nonextinction of all kinds of evolutionary equations. For the following homogeneous Dirichlet boundary value problem:

$$
\begin{gathered}
u_{t}=\Delta u-u^{q}, \quad(x, t) \in \Omega \times(0, \infty), \\
u(x, 0)=u_{0}(x), \quad x \in \Omega, \\
u(x, t)=0, \quad x \in \partial \Omega \times(0, \infty) .
\end{gathered}
$$

$\mathrm{Gu}$ [2] obtained that the nontrivial solutions of the problem (2) vanish identically in a finite time if and only if $0<q<1$, which implies that strong absorption will cause extinction in a finite time. More results on the extinction for the problem (2) have also been obtained by many researchers, and we can refer to [3-7] and the references therein. Because of the occurrence of such a phenomenon for a diffusion equation with a different absorption term, that is, the absorption term is a nonnegative function of $\nabla u$ instead of being a nonnegative function of $u$, Benachour et al. $[8,9]$ considered the following Cauchy problem for the viscous Hamilton-Jacobi equation:

$$
\begin{gathered}
u_{t}=\Delta u-|\nabla u|^{q}, \quad(x, t) \in R^{N} \times(0, \infty), \\
u(x, 0)=u_{0}(x), \quad x \in R^{N} .
\end{gathered}
$$

They proved that the nonnegative classical solutions to the problem (3) are extinct in finite time and have noncompact support if $0<q<1$. 
Generally, in the problems (2) and (3), there is a comparison between the diffusion term and the absorption term, and the absorption is sufficiently strong to lead any bounded nonnegative solution to zero in finite time. However, while both the source $-u^{q}$ in problem (2) and the source $-|\nabla u|^{q}$ in problem (3) are called the "cool source", the nonlinear source $+|\nabla u|^{q}$ in problem (1) is physically called the "hot source." As far as we know, the type of "hot source" has complicated influences on the properties of solutions compared with the case of "cool source" [10]. Thus, few works are concerned with extinction property for solutions to the evolutionary equations with "hot source." In 2005, Li and Wu [10] gave some necessary and sufficient conditions of extinction for the solutions to the following problem with "hot source":

$$
\begin{gathered}
u_{t}=\Delta u^{m}+\lambda u^{p}, \quad(x, t) \in \Omega \times(0, \infty), \\
u(x, 0)=u_{0}(x) \geq 0, \quad x \in \Omega, \\
u(x, t)=0, \quad x \in \partial \Omega \times(0, \infty),
\end{gathered}
$$

where $0<m<1$. They proved that if $p>m$, the solutions to the problem (4) with small initial data vanished in finite time and if $p<m$, the maximal solution to the problem (4) is positive for all $t>0$. Recently, Tian and Mu [11] studied the following $p$-Laplacian equation with nonlinear "hot source":

$$
\begin{gathered}
u_{t}=\operatorname{div}\left(|\nabla u|^{p-2} \nabla u\right)+\lambda u^{p}, \quad(x, t) \in \Omega \times(0, \infty), \\
u(x, 0)=u_{0}(x), \quad x \in \Omega, \\
u(x, t)=0, \quad x \in \partial \Omega \times(0, \infty) .
\end{gathered}
$$

They obtained that for $1<p<2, q=p-1$ is the critical exponent of extinction for the weak solution to the problem (5). Also, they show that for $1<p<2$ and $q=p-1$, the extinction and nonextinction of the solution to the problem (5) depend strongly on the first eigenvalue of the problem $-\operatorname{div}\left(|\nabla u|^{p-2} \nabla u\right)=\lambda|u|^{p-2} u$ in $\Omega,\left.u\right|_{\partial \Omega}=0$. For more results on the extinction properties of equations with "hot source," we can refer to $[12,13]$ and the references therein.

By replacing the diffusion term $u^{p}$ with the gradient absorption terms $|\nabla u|^{p}$ in (4), in this paper, we devote to establish the conditions for the extinction of solution to the problem (1), which involves the "hot source" rather than the "cool source."

\section{Preliminaries}

In this section, we will give some definitions and lemmas which is useful to the proof of our results in the next section. For our convenience, we first define some sets as follows:

$$
\begin{gathered}
\Omega_{T}=\Omega \times(0, T), T>0, \\
E=\left\{u_{t} \in L^{2}\left(\Omega_{T}\right):\right. \\
\left.u \in L^{2 m}\left(\Omega_{T}\right) \bigcap L^{2}\left(\Omega_{T}\right) ; \nabla u \in L^{2 p}\left(\Omega_{T}\right)\right\} \\
E_{0}=\left\{\varphi \in \Omega_{T}: \varphi_{t}, \Delta \varphi ;|\nabla \varphi| \in L^{2}\left(\Omega_{T}\right) ;\left.\varphi\right|_{\partial \Omega_{T}}=0\right\} .
\end{gathered}
$$

It is well known that the problem (1) has no classical solution in general. We need to consider its weak solutions which is defined as follows.

Definition 1. For any $T \geq 0$, a function $u(x, t) \in E$ is called a weak solution to the problem (1) if the following equalities hold for any $0<t_{1}<t_{2}<T$ and $0 \leq \varphi \in E_{0}$ :

$$
\begin{gathered}
\int_{\Omega} u\left(x, t_{2}\right) \varphi\left(x, t_{2}\right) d x-\int_{\Omega} u\left(x, t_{1}\right) \varphi\left(x, t_{1}\right) d x \\
=\int_{t_{1}}^{t_{2}} \int_{\Omega} u \varphi_{t}+u^{m} \Delta \varphi+|\nabla u|^{p} \varphi d t d x, \\
u(x, 0)=u_{0}(x), \quad \text { a.e. } x \in \Omega .
\end{gathered}
$$

Remark 2. Similarly, to define a subsolution (resp., supersolution) $\underline{u}(x, t)$ (resp., $\bar{u}(x, t))$ of the problem (1), we need only to set $\underline{u}(x, 0)<u_{0}(x)$ (resp., $\left.\bar{u}(x, 0)>u_{0}(x)\right)$ in $\Omega, \underline{u}(x, t) \leq 0$ (resp., $\bar{u}(x, t) \geq 0)$ on $\partial \Omega \times(0, \infty)$, and the first equality in (7) is replaced by $\leq$ (resp., $\geq$ ) for every $\varphi(x)>0$.

As a useful tool in the proof of our main results in the next section, a comparison principle of the following problem is needed:

$$
\begin{gathered}
u_{t}=\Delta \varphi(x, t, u)+f(x, t, u), \quad(x, t) \in \Omega \times(0, T), \\
u(x, 0)=u_{0}(x), \quad x \in \Omega, \\
u(x, t)=0, \quad x \in \partial \Omega \times(0, T) .
\end{gathered}
$$

Under the following four hypotheses:

(H1) $\varphi, \Delta_{x} \varphi \in C\left(\overline{\Omega_{T}} \times R\right), \nabla_{x} \varphi \in \prod_{i=1}^{N} C\left(\overline{\Omega_{T}} \times R\right)$, and $\varphi_{u} \in$ $C\left(\overline{\Omega_{T}} \times R \backslash\{0\}\right)$, s.t. $\varphi(x, t, 0)=0$ and $\varphi_{u}(x, t, u)>0$ for all $(x, t) \in \overline{\Omega_{T}}$ and $u \neq 0$;

(H2) $f \in C\left(\overline{\Omega_{T}} \times R\right)$ and $f_{u} \in C\left(\overline{\Omega_{T}} \times R \backslash\{0\}\right)$ with $f(x, t, 0)=0$ for all $(x, t) \in \overline{\Omega_{T}}$;

(H3) $u_{0} \in L^{\infty}(\Omega)$ with $u_{0}>0$;

(H4) for any constant $a>0$, if $u \geq 0, v \geq a$ in $\overline{\Omega_{T}}$ and $u, v \in L^{\infty}\left(\Omega_{T}\right)$, then the functions $\Phi$ and $F$ defined almost everywhere in $\Omega_{T}$ by

$$
\begin{gathered}
\Phi(x, s) \equiv \int_{0}^{1} \varphi_{u}(x, s, \theta u+(1-\theta) v) d \theta \\
F(x, s) \equiv \int_{0}^{1} \varphi_{f}(x, s, \theta u+(1-\theta) v) d \theta
\end{gathered}
$$

belong to $L^{\infty}\left(\Omega_{T}\right)$ and $L^{\infty}\left(\overline{\partial \Omega_{T}} \times[0, T]\right)$, respectively,

we have the following lemma on the comparison principle of the problem (8).

Lemma 3 (see [14]). Assume that the hypotheses (H1)-(H4) hold. Let $u\left(x, t ; u_{0}\right)$ and $v\left(x, t ; v_{0}\right)$ be nonnegative solutions of (8) on $\Omega_{T}$ with $u_{0} \leq v_{0}$. Assume further that for any $t_{1}<T$, there is a constant $a\left(t_{1}\right)>0$ such that $v\left(x, t ; v_{0}\right) \geq a\left(t_{1}\right)$ for all $(x, t) \in \bar{\Omega} \times\left[0, t_{1}\right]$. Then, $u \leq v$ almost everywhere on $\Omega_{T}$. 


\section{Main Results}

In this section, by applying the energy method introduced in [11] and the comparison result in Lemma 3 of the problem (8), we give the main results on the extinction and nonextinction of the solution to the problem (1).

3.1. Extinction of the Solution. In this subsection, we consider the extinction of the solution to the problem (1) and give the conditions for the extinction of the solution to the problem (1).

Theorem 4. Assume that $0<m<1$, and let $u(x, t)$ be a weak solution of the problem (1). If $m<p \leq 2 /(3-m)$, then, for sufficiently small initial data, there exists a finite time $T$, such that

$$
u(x, t) \equiv 0
$$

for all $(x, t) \in \Omega \times(T,+\infty)$.

Proof. First of all, multiplying the first equation of the problem (1) by $u^{s-1}(s>1)$ and integrating over $\Omega$, we can obtain the following crucial equation to our proof:

$$
\begin{aligned}
& \frac{1}{s} \frac{d}{d t} \int_{\Omega} u^{s} d x+\frac{4 m(s-1)}{(m+s-1)^{2}} \int_{\Omega}\left|\nabla u^{(m+s-1) / 2}\right|^{2} d x \\
& \quad=\lambda\left(\frac{m+s-1}{2}\right)^{-p} \int_{\Omega}\left|\nabla u^{(m+s-1) / 2}\right|^{p} u^{((3-s-m) / 2) p+s-1} d x .
\end{aligned}
$$

Then, we prove the theorem by the following two cases.

First, we consider the case of $(N-2) /(N+2) \leq m<1$. Let $s=1+m$ in (11), and we can get by the Hölder inequality and Poincare inequality that

$$
\begin{aligned}
\|u(\cdot, t)\|_{1+m}^{m} & \leq|\Omega|^{(m /(1+m))-((N-2) / 2 N)}\left\|u^{m}(\cdot, t)\right\|_{2 N /(N-2)} \\
& \leq C_{0}|\Omega|^{(m /(1+m))-((N-2) / 2 N)}\left\|\nabla u^{m}(\cdot, t)\right\|_{2},
\end{aligned}
$$

where $C_{0}$ is the Sobolev embedding constant depending only on $p$ and $N$.

Since $p \leq 2 /(3-m), 0<m<1$, it follows from the Young's inequality for any $\varepsilon>0$ that

$$
\begin{aligned}
& \int_{\Omega}\left|\nabla u^{m}(\cdot, t)\right|^{p} u^{p(1-m)+m} d x \\
& \quad \leq \varepsilon\left\|\nabla u^{m}(\cdot, t)\right\|_{2}^{2}+c(\varepsilon)\|u\|_{2(p-p m+m) /(2-p)}^{2(p-p m+m) /(2-p)}
\end{aligned}
$$

Also, since the assumptions $p \leq 2 /(3-m)$ and $0<m<1$ imply that $2(p-p m+m) /(2-p) \leq 1+m$, we can obtain by the Hölder inequality that

$$
\begin{aligned}
& \|u\|_{2(p-p m+m) /(2-p)}^{2(p-p m+m) /(2-p)} \\
& \quad \leq C_{1}|\Omega|^{2(p-p m+m) /((1+m)(2-p))}\|u\|_{1+m}^{2(p-p m+m) /(2-p)} .
\end{aligned}
$$

Therefore, from the previous inequalities (11)-(14), we can obtain the following differential inequality:

$$
\begin{aligned}
\frac{1}{1+m} & \frac{d}{d t}\|u\|_{1+m}^{1+m}+\left(1-\lambda m^{-p} \varepsilon\right) C_{0}^{-2} \\
& \times|\Omega|^{(m /(1+m))-((N-2) / 2 N)}\|u\|_{1+m}^{2 m} \\
\leq & \lambda m^{-p} c(\varepsilon) C_{1}|\Omega|^{(2+m p-3 p) /((2-p)(1+m))} \\
& \times\|u\|_{1+m}^{2(p-p m+m) /(2-p)} .
\end{aligned}
$$

Choose $\varepsilon$ sufficiently small such that $1-\lambda m^{-p} \varepsilon>0$ and initial data $\left\|u_{0}\right\|$ sufficiently small such that

$$
\begin{aligned}
& \left\|u_{0}\right\|_{1+m}^{2(p-m) /(2-p)} \\
& \quad<\lambda^{-1} m^{p} c(\varepsilon)^{-1} C_{1}^{-1}\left(1-\lambda m^{-p} \mathcal{\varepsilon}\right) C_{0}^{-2} \\
& \quad \times|\Omega|^{(m /(1+m))-((N-2) / 2 N)-((2+m p-3 p) /((2-p)(1+m)))} .
\end{aligned}
$$

Thus, we can obtain that

$$
\frac{1}{1+m} \frac{d}{d t}\|u\|_{1+m}^{1+m}+C_{3}\|u\|_{1+m}^{2 m} \leq 0
$$

where $C_{2}=\left(1-\lambda m^{-p} \varepsilon\right) C_{0}^{-2}|\Omega|^{((n-2) / 2 n)-(m /(1+m))}-\lambda m^{-p} c(\varepsilon)$ $C_{1}|\Omega|^{((2+m p-3 p) /((2-p)(1+m)))}\left\|u_{0}\right\|_{1+m}^{2(p-m) /(2-p)}>0$.

By integrating the inequality (17), we can obtain that there exists a finite time $T=\|\| u_{0} \|_{1+m}^{1-m} /(1-m) C_{2}$ such that

$$
\begin{gathered}
\|u\|_{1+m} \leq\left(\left\|u_{0}\right\|_{1+m}^{1-m}-(1-m) C_{2} t\right)^{1 /(1-m)}, \quad t \in(0, T], \\
\|u\|_{1+m}=0, \quad t \in[T,+\infty),
\end{gathered}
$$

which implies that $u(x, t)$ vanishes in finite time $T$.

Next, we consider the second case of $0<m<(N-2) /(N+$ $2)<(N-2) / N$. Let $s=(N / 2)(1-m)>1$ in $(11)$, and we can get by the Poincare inequality that

$$
\begin{aligned}
\|u(\cdot, t)\|_{s}^{(m+s-1) / 2} & =\left\|u^{(m+s-1) / 2}(\cdot, t)\right\|_{2 N /(N-2)} \\
& \leq C_{3}\left\|\nabla u^{(m+s-1) / 2}(\cdot, t)\right\|_{2},
\end{aligned}
$$

where $C_{3}$ is the Sobolev embedding constant depending only on $N, m$, and $s$. Also, by the similar calculations as the proof in the first case, we can get from the Young's inequality for any $\eta>0$ that

$$
\begin{aligned}
\int_{\Omega}\left|\nabla u^{(m+s-1) / 2}\right|^{p} u^{((3-s-m) / 2) p+s-1} d x \\
\leq \eta\left\|\nabla u^{(m+s-1) / 2}\right\|_{2}^{2}+c(\eta)|\Omega|^{(2+p m-3 p) /(2-p) s} \\
\quad \times\|u\|_{s}^{((3-s-m) p+2 s-2) /(2-p)} .
\end{aligned}
$$


Choose $\eta$ sufficiently small such that $4 m(s-1) /(m+s-$ $1)^{2}-\lambda(((1+m)((N / 2)-1)) / 2)^{-p} \eta>0$ and initial data $\left\|u_{0}\right\|$ sufficiently small such that

$$
\begin{aligned}
\left\|u_{0}\right\|_{s}^{2(p-m) /(2-p)} & \\
< & \lambda^{-1} c(\eta)^{-1} C_{3}^{-2}\left(\frac{(1+m)((N / 2)-1)}{2}\right)^{-p} \\
& \times|\Omega|^{(2+p m-3 p) /(2-p) s} \\
& \times\left(\frac{4 m(s-1)}{(m+s-1)^{2}}-\lambda\left(\frac{(1+m)((N / 2)-1)}{2}\right)^{-p} \eta\right) .
\end{aligned}
$$

Then, it follows from the inequalities (11) and (19)-(21) that

$$
\frac{1}{s} \frac{d}{d t}\|u\|_{s}^{s}+C_{4}\|u\|_{s}^{m+s-1} \leq 0,
$$

where $C_{4}=C_{3}^{-2}\left(\left(4 m(s-1) /(m+s-1)^{2}\right)-\lambda(((1+m)((N / 2)-\right.$ $\left.1)) / 2)^{-p} \eta\right)-\lambda c(\eta)|\Omega|^{(2+p m-3 p) /(p-2) s}\left\|u_{0}\right\|_{s}^{2(p-m) /(2-p)}$.

By integrating the inequality (22), we can obtain that there exists a finite time $T=\left\|u_{0}\right\|_{s}^{1-m} /(1-m) C_{4}$ such that

$$
\begin{gathered}
\|u\|_{s} \leq\left(\left\|u_{0}\right\|_{s}^{1-m}-(1-m) C_{4} t\right)^{1 /(1-m)}, \quad t \in(0, T] \\
\|u\|_{s}=0, \quad t \in[T,+\infty),
\end{gathered}
$$

which implies that $u(x, t)$ vanishes in finite time $T$. This completes the proof of Theorem 4 .

Theorem 5. If $m=p \leq 2 /(3-m)$, the solution to the problem (1) vanishes in finite time for $\lambda$ sufficiently small.

Proof. When $p=m$, we note that the left sides of the inequalities (16) and (21) always equal 1 . Thus, by a similar argument in the proof of Theorem 4 , we can choose $\lambda$ sufficiently small such that the inequalities (16) and (21) hold, which imply that there exist $T<+\infty$ such that $u(x, t)$ vanishes identically for all $(x, t) \in \Omega_{T}$. This completes the proof of Theorem 5 .

3.2. Nonextinction of the Solution. In this subsection, we investigate the conditions under which the solution $u(x, t)$ of the problem (1) cannot become extinct.

Theorem 6. If $p<m$, the weak solution $u(x, t)$ of (1) cannot vanish in finite time for any nonnegative initial date $u_{0}$ with $\lambda$ being sufficiently large.

Proof. In order to prove Theorem 6, we first define two useful functions as follows. The first function $\phi(x) \in H_{0}^{1}(\Omega)$ satisfying $\max _{x \in \Omega} \phi(x)=1$ is the associated eigenfunction with the principal eigenvalue $\lambda_{1}$ of the following problem:

$$
\begin{gathered}
-\Delta \phi=\lambda_{1} \phi, \quad x \in \Omega, \\
\left.\phi\right|_{\partial \Omega}=0 .
\end{gathered}
$$

For $p<m$, we define the second useful function as follows:

$$
g(t)=\left(\frac{a}{b}\right)^{1 /(m-p)}(1-\exp (-C t))^{1 /(1-p)}
$$

where $a<b$ and $C \in\left(0,(m-p)\left(a^{1-p} / b^{1-m}\right)^{1 /(1-p)}\right)$. It follows from [11] that the function $g(t)$ satisfies the following properties:

$$
\begin{gathered}
g^{\prime}(t) \leq-a g^{m}(t)+b g^{p}(t), \\
g(0)=0, \\
0<g(t)<1, \quad \text { for } t>0 .
\end{gathered}
$$

Now, let $v(x, t)=g(t) \phi(x)^{1 / m}$ be a function $\Omega \times(0, \infty)$. Next, we will show that $v(x, t)$ is a subsolution of the problem (1). In fact, from the definitions of functions $g(t), v(x, t)$ and the properties (26) of the function $g(t)$, we have that

$L(v(x, t))$

$$
\begin{aligned}
& =\int_{0}^{t} \int_{\Omega} v_{t}(x, s) \varphi(x, s) d x d s \\
& +\int_{0}^{t} \int_{\Omega} \nabla u^{m} \cdot \nabla \varphi-\lambda|\nabla v|^{p} \varphi(x, s) d x d s \\
& =\int_{0}^{t} \int_{\Omega}\left(v_{t}(x, s)-\Delta v^{m}-|\nabla v|^{p}\right) \varphi(x, s) d x d s \\
& =\int_{0}^{t} \int_{\Omega}\left[g^{\prime}(t) \phi(x)^{1 / m}+\lambda_{1} g^{m}(t) \phi(x)\right. \\
& \left.-\lambda g^{p}(t)\left(m^{-p} \phi(x)^{((1-m) p) / m}|\nabla \phi|^{p}\right)\right] \\
& \times \varphi(x, s) d x d s \\
& \leq \int_{0}^{t} \int_{\Omega}\left\{-a g^{m}(t) \phi(x)^{1 / m}-\lambda g^{p}(t)\right. \\
& \times\left[m^{-p} \phi(x)^{((1-m) p) / m}|\nabla \phi|^{p}\right. \\
& \left.\left.-\frac{b}{\lambda} \phi(x)^{1 / m}-\frac{\lambda_{1}}{\lambda} g^{m-p}(t) \phi(x)\right]\right\} \\
& \times \varphi(x, s) d x d s
\end{aligned}
$$

$$
\begin{aligned}
\leq \int_{0}^{t} \int_{\Omega}-\lambda g^{p}(t)[ & m^{-p} \phi(x)^{((1-m) p) / m}|\nabla \phi|^{p} \\
& \left.-\frac{b}{\lambda} \phi(x)^{1 / m}-\frac{\lambda_{1}}{\lambda} g^{m-p}(t) \phi(x)\right]
\end{aligned}
$$

$\times \varphi(x, s) d x d s$ 


$$
\begin{aligned}
\leq \int_{0}^{t} \int_{\Omega} & -\lambda g^{p}(t) \phi(x)^{((1-m) p) / m} \\
\times & {\left[m^{-p}|\nabla \phi|^{p}-\frac{b}{\lambda} \phi(x)^{(1-p+p m) / m}\right.} \\
& \left.\quad-\frac{\lambda_{1}}{\lambda} g^{m-p}(t) \phi(x)^{(m-p+p m) / m}\right] \varphi(x, s) d x d s .
\end{aligned}
$$

In order to prove that $L(v(x, t))<0$ which implies that $v(x, t)$ is a subsolution of the problem (1), we only show that

$$
\begin{aligned}
\int_{\Omega} m^{-p} & |\nabla \phi|^{p}-\frac{b}{\lambda} \phi(x)^{(1-p+p m) / m} \\
& -\frac{\lambda_{1}}{\lambda} g^{m-p}(t) \phi(x)^{(m-p+p m) / m} d x \geq 0 .
\end{aligned}
$$

Since $0<g(t)<1$ and $m>p$, we have that

$$
\begin{gathered}
g^{m-p}(t)<1, \\
\phi(x)^{(1-p+p m) / m}<\phi(x)^{(m-p+p m) / m} .
\end{gathered}
$$

By choosing $\lambda \geq m^{p}\left(b+\lambda_{1}\right)\left(\|\phi\|_{(m-p+p m) / m}^{(m-p+p m) / m} /\|\nabla \phi\|_{p}^{p}\right)$, we can get that

$$
\int_{\Omega} m^{-p}|\nabla \phi|^{p}-\frac{b+\lambda_{1}}{\lambda} \phi(x)^{(m-p+p m) / m} d x \geq 0,
$$

which together with (29) implies that (28) holds. Therefore, $v(x, t)$ is a subsolution of the problem (1). Moreover, since $v(x, 0)=g(0) \phi(x)=0 \leq u_{0}$ in $\Omega$ and $\left.v\right|_{(\partial \Omega)_{t}}=0$, we can obtain by the comparison principle that $u(x, t) \geq v(x, t)>0$ in $\Omega \times(0,+\infty)$, which implies that the weak solution $u(x, t)$ of (1) cannot vanish in finite time. This completes the proof of Theorem 6.

Remark 7. From Theorems 4-6, we observe that $q=m$ is the critical exponent of extinction for the solution to the problem (1).

\section{Acknowledgments}

This work is supported in part by NSF of China (11101069, 11071266) and in part by the Natural Science Foundation Project of CQ CSTC (2010BB9218).

\section{References}

[1] A. S. Kalashnikov, "The nature of the propagation of perturbations in problems of nonlinear heat conduction with absorption," USSR Computational Mathematics and Mathematical Physics, vol. 14, no. 4, pp. 70-85, 1974.

[2] Y. G. Gu, "Necessary and sufficient conditions for extinction of solutions to parabolic equations," Acta Mathematica Sinica, vol. 37, no. 1, pp. 73-79, 1994 (Chinese).

[3] E. Dibenedetto, Degenerate Parabolic Equations, Springer, New York, NY, USA, 1993.
[4] L. C. Evans and B. F. Knerr, "Instantaneous shrinking of the support of nonnegative solutions to certain nonlinear parabolic equations and variational inequalities," Illinois Journal of Mathematics, vol. 23, no. 1, pp. 153-166, 1979.

[5] A. Friedman and M. A. Herrero, "Extinction properties of semilinear heat equations with strong absorption," Journal of Mathematical Analysis and Applications, vol. 124, no. 2, pp. 530546, 1987.

[6] V. A. Galaktionov and J. L. Vazquez, "Continuation of blowup solutions of nonlinear heat equations in several space dimensions," Communications on Pure and Applied Mathematics, vol. 50, no. 1, pp. 1-67, 1997.

[7] M. A. Herrero and J. J. L. Velázquez, "Approaching an extinction point in one-dimensional semilinear heat equations with strong absorption," Journal of Mathematical Analysis and Applications, vol. 170, no. 2, pp. 353-381, 1992.

[8] S. Benachour, Ph. Laurençot, and D. Schmitt, "Extinction and decay estimates for viscous Hamilton-Jacobi equations in $R^{N}$," Proceedings of the American Mathematical Society, vol. 130, no. 4, pp. 1103-1111, 2002.

[9] S. Benachour, Ph. Laurençot, D. Schmitt, and Ph. Souplet, "Extinction and non-extinction for viscous Hamilton-Jacobi equations in $R^{N}$," Asymptotic Analysis, vol. 31, no. 3-4, pp. 229-246, 2002.

[10] Y. Li and J. Wu, "Extinction for fast diffusion equations with nonlinear sources," Electronic Journal of Differential Equations, vol. 2005, no. 23, pp. 1-7, 2005.

[11] Y. Tian and C. Mu, "Extinction and non-extinction for a $p$ Laplacian equation with nonlinear source," Nonlinear Analysis: Theory, Methods \& Applications A, vol. 69, no. 8, pp. 2422-2431, 2008.

[12] W. Liu and B. Wu, "A note on extinction for fast diffusive $p$ Laplacian with sources," Mathematical Methods in the Applied Sciences, vol. 31, no. 12, pp. 1383-1386, 2008.

[13] J. Yin and C. Jin, "Critical extinction and blow-up exponents for fast diffusive $p$-Laplacian with sources," Mathematical Methods in the Applied Sciences, vol. 30, no. 10, pp. 1147-1167, 2007.

[14] A. W. Leung and Q. Zhang, "Finite extinction time for nonlinear parabolic equations with nonlinear mixed boundary data," Nonlinear Analysis: Theory, Methods \& Applications A, vol. 31, no. 1-2, pp. 1-13, 1998. 


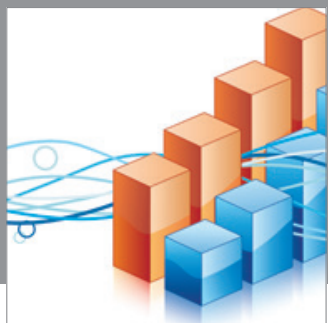

Advances in

Operations Research

mansans

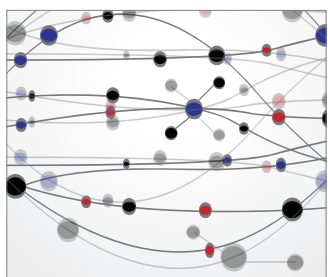

The Scientific World Journal
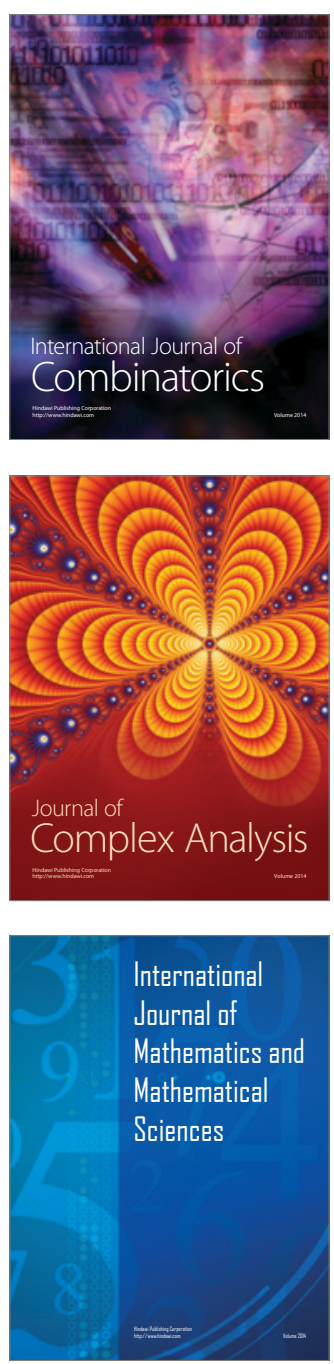
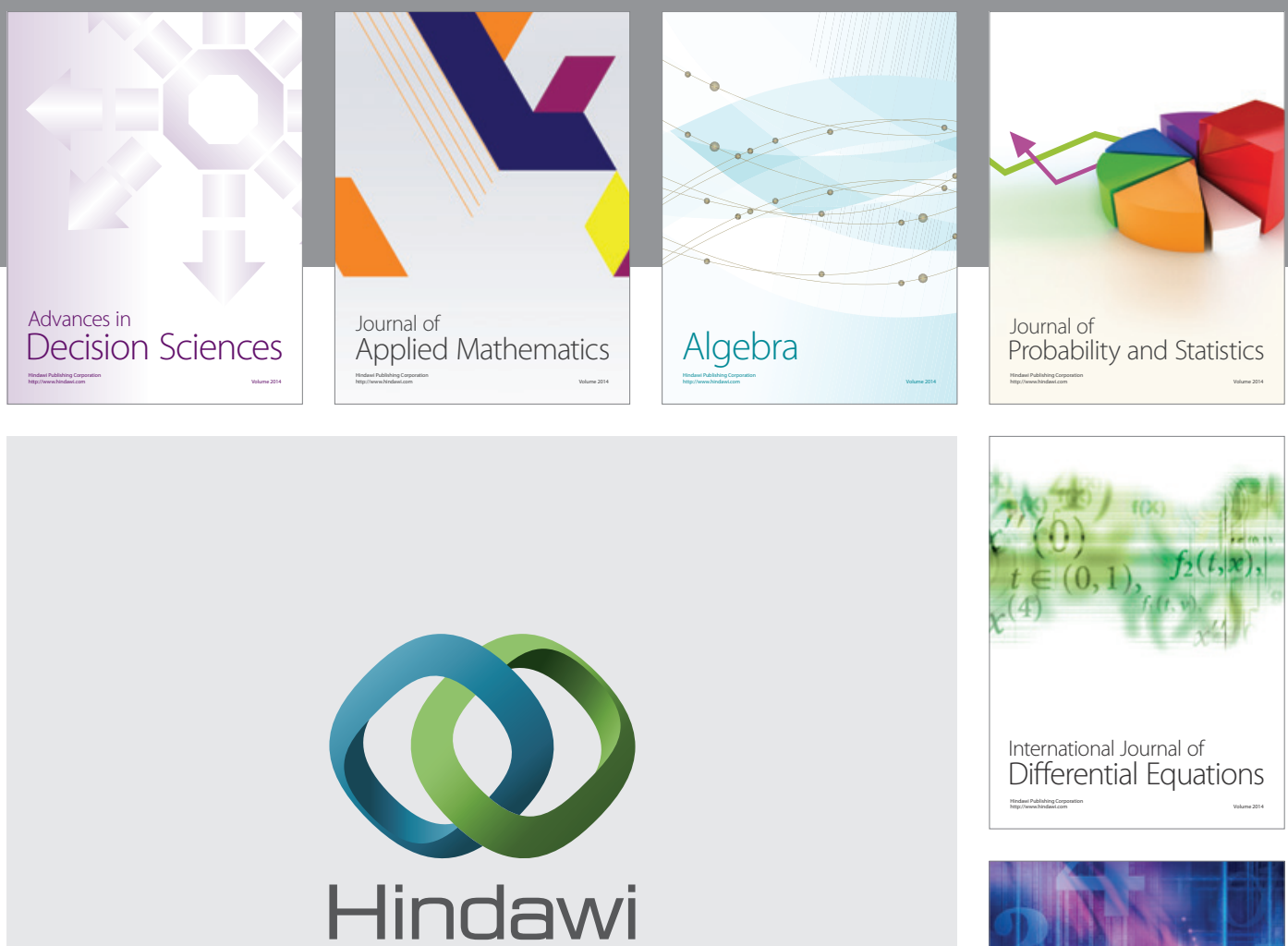

Submit your manuscripts at http://www.hindawi.com
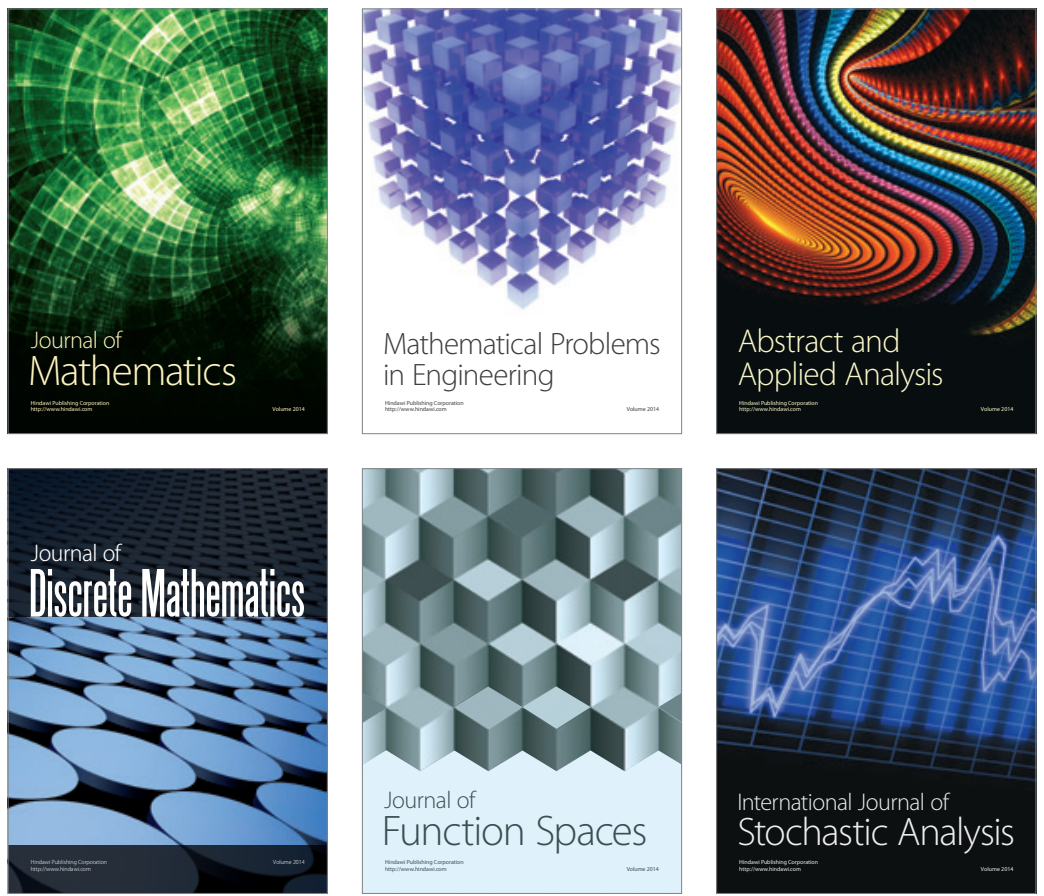

Journal of

Function Spaces

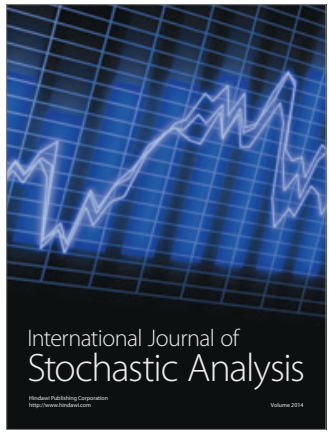

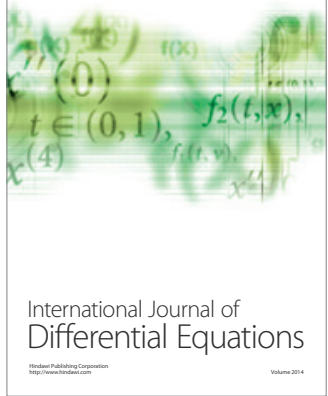
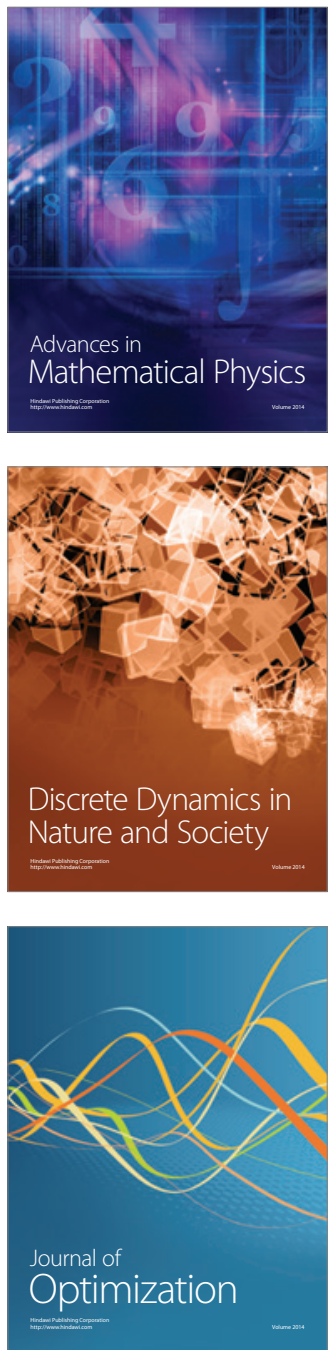